\title{
Does epidural analgesia modify the risk of complications after gastrectomy?
}

\author{
Tomasz Olesiński', Marta Urbańska', Anna Borkowska², Paulina Wieszczy ${ }^{1,3}$, Dariusz Król', \\ Dorota Rucz ${ }^{4}$, Małgorzata Symonides ${ }^{1}$
}

\author{
'Maria Sklodowska-Curie National Research Institute of Oncology, Warsaw, Poland \\ ${ }^{2}$ City Hospital Czerniakowski, Warsaw, Poland \\ ${ }^{3}$ Medical Centre for Postgraduate Education, Warsaw, Poland \\ ${ }^{4}$ County Health Center, Otwock, Poland
}

Introduction. The surgical treatment of Gastric Cancer is associated with overall complication rates as high as $50 \%$. The intent of this study was to assess the impact of epidural analgesia (EA) on postoperative complication rates among patients undergoing gastric resections.

Materials and methods. Of the 617 gastric cancer patients who between 2002 and 2010 had undergone stomach resection, 246 (39.8\%) were administered EA. Groups with and without EA were compared.

Results. The general rate of complications was lower in the EA group in the univariable analysis - 38.5\% vs. 54.2\% (odds ratio [OR]: 0.47, 95\% confidence interval [Cl]: 0.34-0.66, $p<0.001$ ), intra-abdominal abscess (OR 0,28, 95\% Cl: 0.14-0.59, $p=0.001)$, pneumonia (OR 0,39, 95\% Cl: 0.24-0.63, p<0.001), temperature $>38^{\circ} \mathrm{C}(\mathrm{OR} 0.53,95 \% \mathrm{Cl}: 0.37-0.74, \mathrm{p}<0.001)$ and re-operation (OR 0.53, 95\% Cl: 0.28-1.00, $\mathrm{p}=0.049$ ). These relationships were confirmed in a multivariable analysis for the general number of complications (OR 0.53, 95\% Cl: 0.37-0.75, p < 0.001), intra-abdominal abscess (OR 0.36, 95\% Cl: 0.16-0.77, p=0.009), temperature $>38^{\circ} \mathrm{C}$ (OR 0.56, 95\% Cl: 0.39-0.82, $\mathrm{p}=0.009$ ), pneumonia (OR 0.42, 95\% Cl: $\left.0.25-0.71, \mathrm{p}=0.001\right)$.

Conclusions. Our findings indicate that postoperative treatment with EA for patients undergoing stomach resection is safe and contributes to a reduction in the number of postoperative complications.

Key words: gastric cancer, GEJ cancer, epidural analgesia, postoperative complications, gastrectomy, postoperative pain

\section{Introduction}

For the past 100 years, cases of stomach cancer (gastric cancer - GC) amongst developed countries have been systemically in decline. Possible contributing factors for this decline may be attributed to the increased use of refrigeration for food storage, dietary changes, and decreased incidents of infections with Helicobacter pylori [1]. Despite progress, stomach cancer remains the fourth most frequently diagnosed cancer worldwide. In 2008, there were 980,000 new cases, of which, 83,000 were reported in the European Union and over 5000 in Poland alone [1, 2]. In Poland, the 5 -year survival rate post stomach cancer diagnosis is about $18 \%$, in Europe is about 25\%, while in Japan about 70\% [2-4].

Surgical resection of gastric cancer has produced suboptimal survival rates despite multidisciplinary treatment appro-

\section{How to cite:}

Olesiński T, Urbańska M, Borkowska A, Wieszczy P, Król D, Rucz D, Symonides M. Does epidural analgesia modify the risk of complications after gastrectomy? NOWOTWORY J OnCol 2021; 71: 202-211

This article is available in open access under Creative Common Attribution-Non-Commercial-No Derivatives 4.0 International (CC BY-NC-ND 4.0) license, allowing to download articles and share them with others as long as they credit the authors and the publisher, but without permission to change them in any way or use them commercially. 
aches and improvements in surgical techniques. The European Society for Medical Oncology (ESMO) guidelines of treatment for patients diagnosed with an advanced GC include perioperative chemotherapy [5]. However, a total or subtotal gastrectomy with removal of the surrounding lymph nodes, (D2 resection) remains the only curative method of treatment [5-8]. The vast extent of surgical intervention is one of the main contributing factors to the high risk of complications associated with the procedure. The estimated number of complications varies between 17 and 48\%. Additionally, gastric resection in conjunction with splenectomy or spleno-pancreatectomy significantly increases the potential for complications [9-14]. The management of quality care in postsurgical settings that include administration of regional analgesia contributes to better treatment outcomes $[15,16]$. Due to the limitations in the use of opioids, resulting from the recommendations of the ERAS protocol, the effectiveness of epidural analgesia (EA) is very important. Currently, EA is a standard procedure in our team and for this reason historical data were compared. On the other hand, surgical procedures did not undergo significant modification during the period under review.

\section{Objective}

To assess the impact of EA on postoperative complication rates in patients undergoing subtotal or total gastrectomy for gastric cancer.

\section{Materials and methods}

This study was conducted in a single institution using its administrative database. All patients were treated between 2002 and 2010 at The Maria Sklodowska-Curie Institute, Oncology Center in Warsaw. No neoadjuvant therapy was administered in the analyzed period. Of $723 \mathrm{GC}$ resections performed, 617 cases had complete medical documentation that was adequate for retrospective review (study flow - fig. 1).

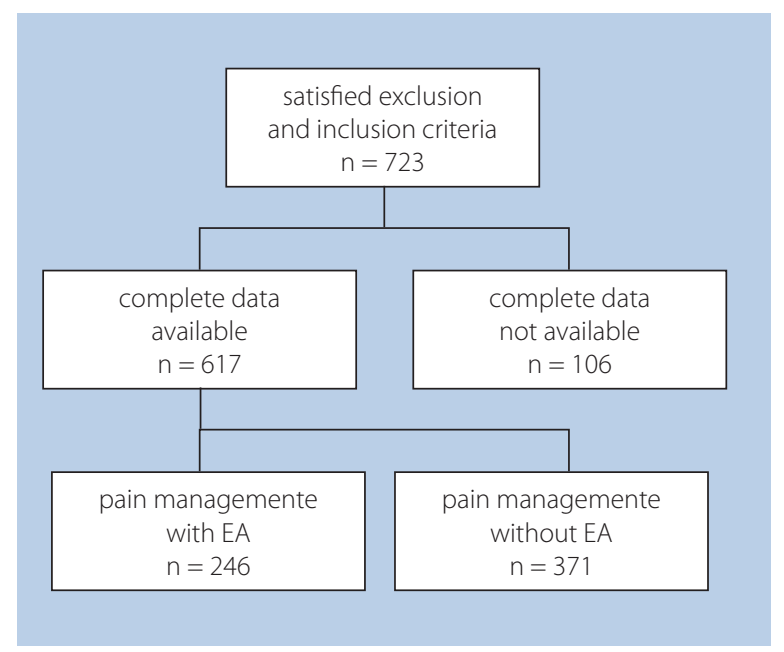

Figure 1. Flow-chart of the study
Table I. Baseline characteristics - demographic data, nutrition status, and comorbidities

\begin{tabular}{|c|c|c|c|}
\hline \multirow[t]{2}{*}{ Characteristic } & \multicolumn{2}{|c|}{ Epidural analgesia (EA) } & \multirow[t]{2}{*}{ p value } \\
\hline & $\begin{array}{c}\text { No } \\
n=371(\%)\end{array}$ & $\begin{array}{c}\text { Yes } \\
n=246(\%)\end{array}$ & \\
\hline $\begin{array}{l}\text { gender: } \\
\text { - female } \\
\text { - male }\end{array}$ & $\begin{array}{l}119(58.3) \\
252(61)\end{array}$ & $\begin{array}{l}85(41.7) \\
161(39)\end{array}$ & 0.522 \\
\hline $\begin{array}{l}\text { age: (median) } \\
\text { (IQR) }\end{array}$ & $\begin{array}{c}64 \\
(53-71)\end{array}$ & $\begin{array}{c}61 \\
(54-72)\end{array}$ & 0.144 \\
\hline $\begin{array}{l}\text { BMI } \\
\cdot \quad<19 \\
\cdot \quad 19-25 \\
\cdot \quad>25\end{array}$ & $\begin{array}{c}31(70.5) \\
164(63.8) \\
176(55.7)\end{array}$ & $\begin{array}{c}13(29.5) \\
93(36.2) \\
140(44.3)\end{array}$ & 0.050 \\
\hline comorbidities & $296(61.0)$ & $189(39.0)$ & 0.381 \\
\hline diabetes & $28(54.9)$ & $23(45.1)$ & 0.426 \\
\hline coronary disease & $107(60.1)$ & $71(39.9)$ & 0.996 \\
\hline hypertension & $125(53.6)$ & $108(46.4)$ & 0.010 \\
\hline peptic ulcer & $93(64.1)$ & $52(35.9)$ & 0.260 \\
\hline anemia & $304(58.6)$ & $215(41.4)$ & 0.069 \\
\hline
\end{tabular}

EA - epidural analgesia; IQR - interquartile range

The data of 617 patients diagnosed with gastric cancer that underwent resection of the stomach was retrospectively reviewed and analyzed by univariable and multivariable methods. Patients were divided into two study groups based on the use of epidural analgesia and other methods. The group of patients treated without EA included patients who underwent treatment during a period of time when epidural catheterization use was not the treatment of choice (until the end of 2006); these cases primarily occurred historically earlier than those who were treated with EA. Another reason for non EA administration was the lack of patient consent. Our study included 413 males (66.9\%) and 204 females (33.1\%) with a median age of 63 (53-71). Epidural analgesia was administered in 246 patients (39.8\%). The patients' demographic and clinical characteristics are illustrated in table I and table II.

Analysis of the two group of patients indicated differences in the location of the gastric tumor, the extent of the gastric resection, and spleen removal. Patients treated without EA more frequently experienced malnutrition (BMI < 19). We did not observe statistically significant differences among both studied groups in respect to demographic characteristics, pre-operative risk factors (excluding hypertension), and the length of the procedure. The statistical univariable and multivariable analysis of the factors contributing to postsurgical complications included:

- administered EA,

- gender,

- age,

- pre-surgical BMI,

- diabetes,

- hypertension, 
Table II. Type and extent of surgical intervention

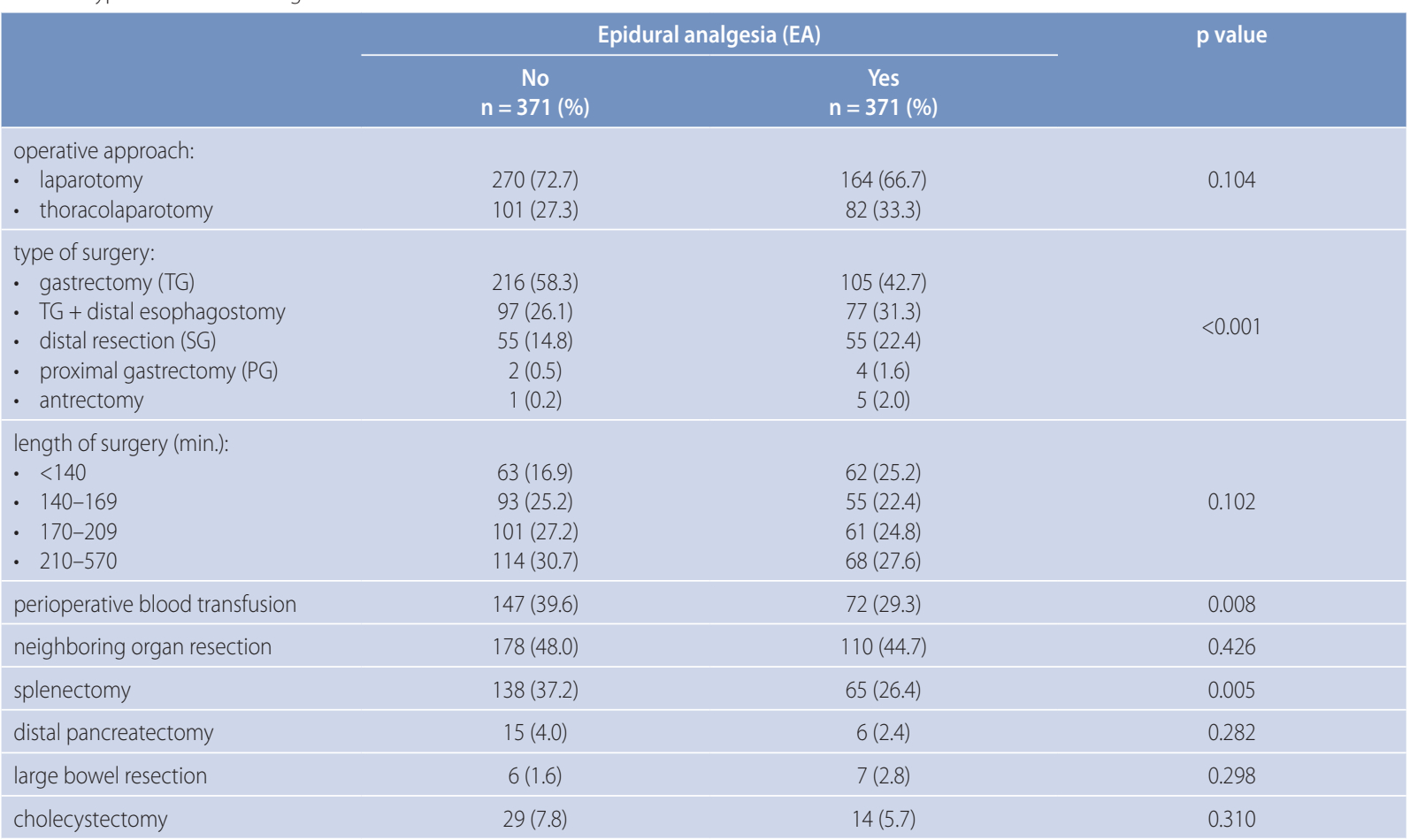

- coronary disease,

- and peptic ulcers.

Additionally, in our analysis we included perioperative transfusions, the length of the surgery, and the extent of the multi-organ resection.

\section{Operative treatment}

All cases included in this study contained patients who were operated on by the same experienced (over 30 operations per surgeon) surgical team. Post-operative care and management was provided using consistent post-surgical protocols that included enteral and parenteral nutrition for a period of 7 to 10 days. Total gastrectomy (TG) was performed on 321 patients (52\%), 174 (28.2\%) patients were treated with TG expanded by resection of the lower section of the esophagus, $110(17.85 \%)$ patients underwent distal subtotal gastrectomy (SG), and in 6 (1\%) of these cases proximal gastrectomy (PG) was performed. In 434 (70.3\%) of these cases, surgery was performed by laparotomy, in 183 (29.7\%) patient's a laparotomy was performed via the thoraco-abdominal approach. We performed curative gastrectomies and dissections of the lymph nodes expanded by removal of the additional organs in cases rendering more extensive surgical interventions. The range of surgical resections in both groups of patients is presented in table II.

\section{Postoperative pain management}

All patients (from 2007) were preoperatively evaluated for the postoperative use of epidural analgesia. Additionally, patients that were administered epidural analgesia consented to the procedure in a separate preoperative assessment. We administered EA in all suitable cases, except in patients with clinical contraindications to the procedure, and in cases where the patient did not consent. Contraindications included:

- coagulation disorders or perioperative use of blood clotting medications,

- inflammation at the catheter placemat area,

- neurological conditions.

Prior to administering general anesthesia, in the operating room, the epidural catheter was placed into the epidural space between Th6 and Th7 (when the patient's anatomy dictated, exact vertebral space varied by one up/down segments). The area designated for catheter placement was prepared according to surgical protocols, with the insertion site disinfected and surgical dressing administered. The skin and the subcutaneous tissue in the puncture site was anesthetized with a $2 \%$ solution of lidocaine and kept sterile. After the catheter was inserted into the epidural space, it was secured on the skin surface with clearly marked transparent dressing tape. Our postoperative pain management regimen of choice was epidural analgesia, administered via continuous infusion of Breivik's mixture into the epidural space using a syringe pump [17]. The mixture was composed of low concentrations of medications ( $22 \mu \mathrm{g} / \mathrm{ml}$ adrenaline, $2 \mu \mathrm{g} / \mathrm{ml}$ fentanyl and $1.25 \mathrm{mg} / \mathrm{ml}$ bupivacaine - which deviates slightly from the standard regimen) in a $0.9 \%$ solution of sodium chloride. The epidural infusion delivery rate was about 3:9 ml/h. Patients in both studied groups were intravenously administered coanal- 
gesics (metamizol and paracetamol). Patients that were not postoperatively administered EA received a subcutaneously delivered morphine sulfate in fractionated doses $(5-10 \mathrm{mg}$ / dose) in 4-6 hour intervals, accompanied with coanalgesics.

\section{Statistical analysis}

Information collected throughout our research was recorded, analyzed, and presented in tables with a cross-tabulation of data. The operative time and age are divided into four categories based on quartiles. The $\mathrm{Chi}^{2}$ test and Wilcoxon test were used to compare the groups. The relationship between postoperative complications (outcomes) and the use of epidural analgesia is analyzed in a univariable logistic regression model and in a multivariable logistic regression model that controls for confounders. Multi-step forward regression was used to select significant disturbing variables in multivariate models, including significant variables at $<0.1$ (the multiple variables describing the EA was included in each model regardless of its significance level). The results of the models are presented in the form of odds ratios (OR) and 95\% confidence intervals (CI). Variables for which $p<0.05$ were considered significant. This analysis is performed with Stata software, version 13.1 (Stata Corporation, College Station, Texas, USA).

\section{Bioethics}

The study was conducted in compliance with the Declaration of Helsinki for medical research and was approved by the Local Bioethics Committee at The Maria Sklodowska-Curie Institute, Oncology Center in Warsaw (permit No. 20/2017 from 09.02.2017). As a retrospective study, according to the approval of the bioethical committee, the informed consent of the patient was not required.

\section{Results}

There were no EA-related complications (neurological deficits, postdural puncture headache), although not every patient managed to insert an epidural catheter. Patients who did not have an epidural catheter inserted for technical reasons were analyzed in the group without EA. Administration of epidural catheters or epidural analgesia is not associated with increased risk for postoperative complications. The postoperative mortality rate was 1\%, (6 patients of 617). No thromboembolic or pulmonary complications were present amongst postoperative patients who had received EA. Due to an insignificant occurrence rate, we did not review incidents of hemoperitoneum (intra-abdominal leak), postoperative eventration, or cases of anastomotic strictures (tab. III).

Additionally, the univariable analysis of patients that were administered EA displayed a lower frequency of postoperative complications compared to the group treated with other methods (OR 0.47, 95\% Cl: 0.34-0.66, p < 0.001), intra-abdominal abscesses (OR 0.28, 95\% Cl: 0.14-0.59, $p=0.001$ ), pneumonia (OR 0.39, 95\% Cl: $0.24-0.63, \mathrm{p}<0.001)$, temperature $>38^{\circ} \mathrm{C}(\mathrm{OR}$
Table III. Postoperative complications

\begin{tabular}{|lcc|}
\hline Complication type & \multicolumn{2}{c}{ Epidural analgesia (EA) } \\
\cline { 2 - 3 } & No & Yes \\
& $n=371(\%)$ & $\mathbf{n}=246(\%)$ \\
\hline overall complications (total) & $201(54.2)$ & $88(35.8)$ \\
\hline temperature $>38^{\circ} \mathrm{C}$ & $158(42.6)$ & $69(28)$ \\
\hline pneumonia & $81(21.8)$ & $24(9.8)$ \\
\hline intra-abdominal abscess & $44(11.9)$ & $9(3.7)$ \\
\hline anastomotic leak & $40(10.8)$ & $20(8.1)$ \\
\hline re-operation & $38(10.2)$ & $14(5.7)$ \\
\hline wound infections & $28(7.5)$ & $13(5.3)$ \\
\hline catheter related sepsis & $28(7.5)$ & $17(6.9)$ \\
\hline anatomic stricture & $6(1.6)$ & $1(0,4)$ \\
\hline intra-abdominal bleeding & $4(1.1)$ & $4(1.6)$ \\
\hline eventration & $2(0.5)$ & $1(0.4)$
\end{tabular}

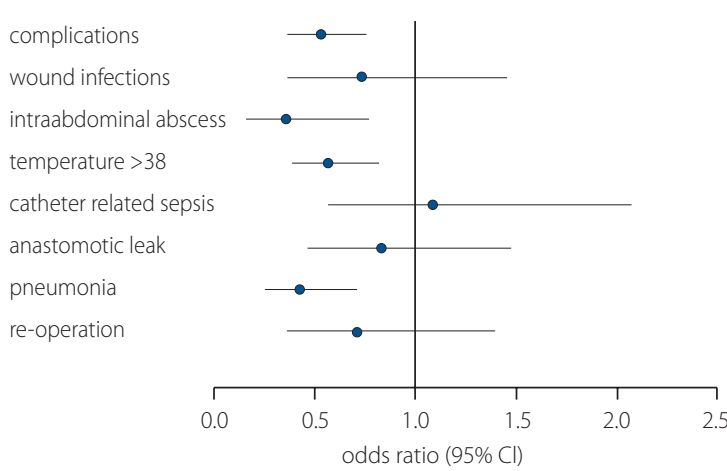

Figure 2. Risk of complications according to EA administration

0.53, 95\% Cl: 0.37-0.74, p < 0.001) and reoperations (OR 0.53, $95 \%$ Cl: $0.28-1.00, p=0.049$ ) (fig. 2).

These relationships were confirmed in a multivariable analysis for the general number of complications (OR 0.53, 95\% Cl: $0.37-0.75, p<0.001)$, intra-abdominal abscess (OR 0.36, 95\% $\mathrm{Cl}: 0.16-0.77, \mathrm{p}=0.009)$, temperature $>38^{\circ} \mathrm{C}(\mathrm{OR} 0.56,95 \% \mathrm{Cl}$ : $0.39-0.82, p=0.009$ ), pneumonia (OR 0.42, 95\% Cl: 0.25-0.71, $\mathrm{p}=0.001)$ - tables: IV, V and VI. The relationship between administering EA and reoperation in a multivariable analysis was not confirmed. Diagnosis of pneumonia was based on the correlation of clinical symptoms and radiological determinations. There were no significant statistical differences in univariable and multivariable analysis of wound infections, infection of the central line, or the anastomotic stricture, (table VI and table VII).

\section{Discussion}

Complications associated with gastrectomy with D2 lymphadenectomy for the treatment of gastric cancer presents multiple clinical considerations against extensive lymphadenectomy $[10,13,14]$. The overall rate of complications is between 17 to $48 \%$. The most frequent postoperative complications in gastric resection surgeries for curative gastric 


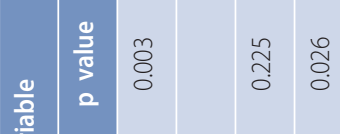

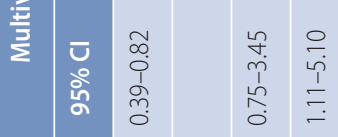

ণ

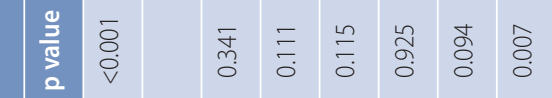

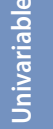

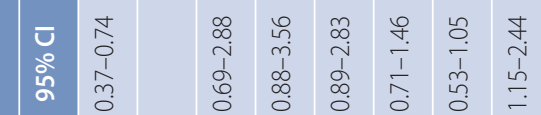

ษั.

$\frac{\sqrt{2}}{\frac{\pi}{\tilde{2}}}$

๓

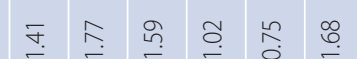

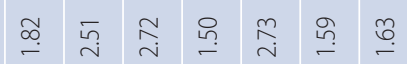

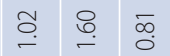

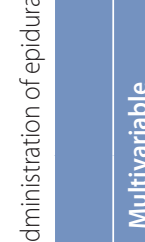

学

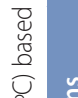

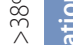

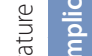

总

垔

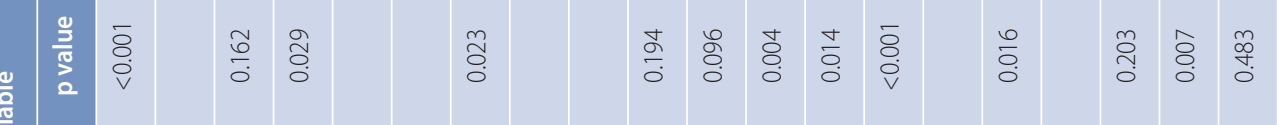

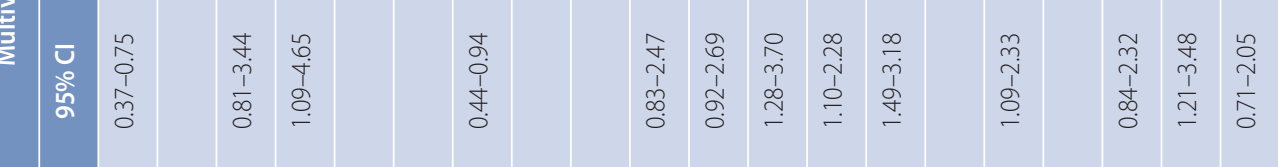

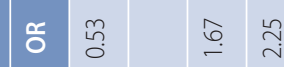

đ̊.

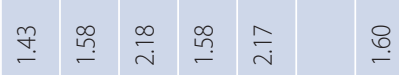

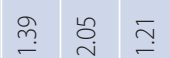

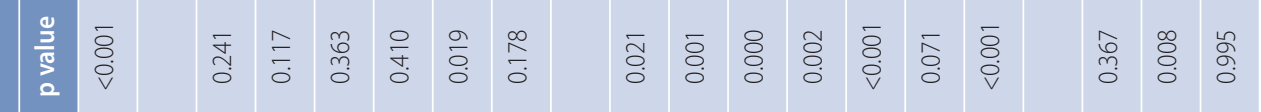
竞

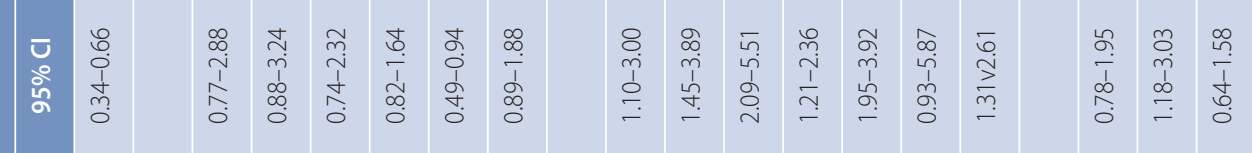

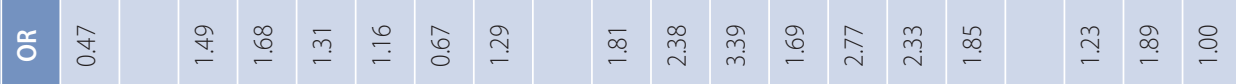

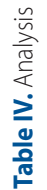




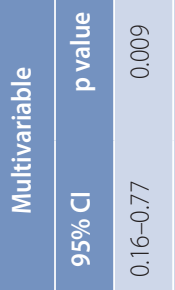

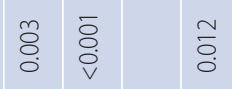

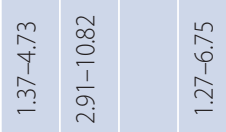

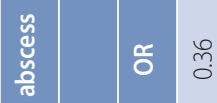

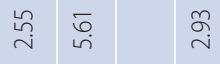

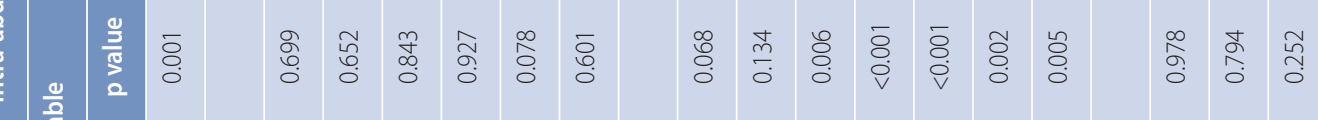

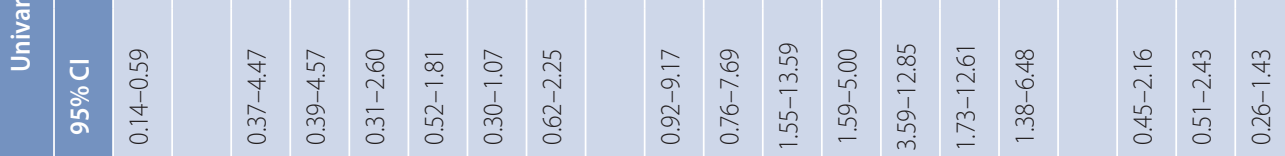

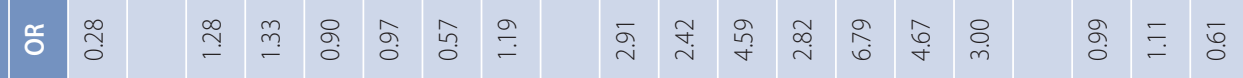

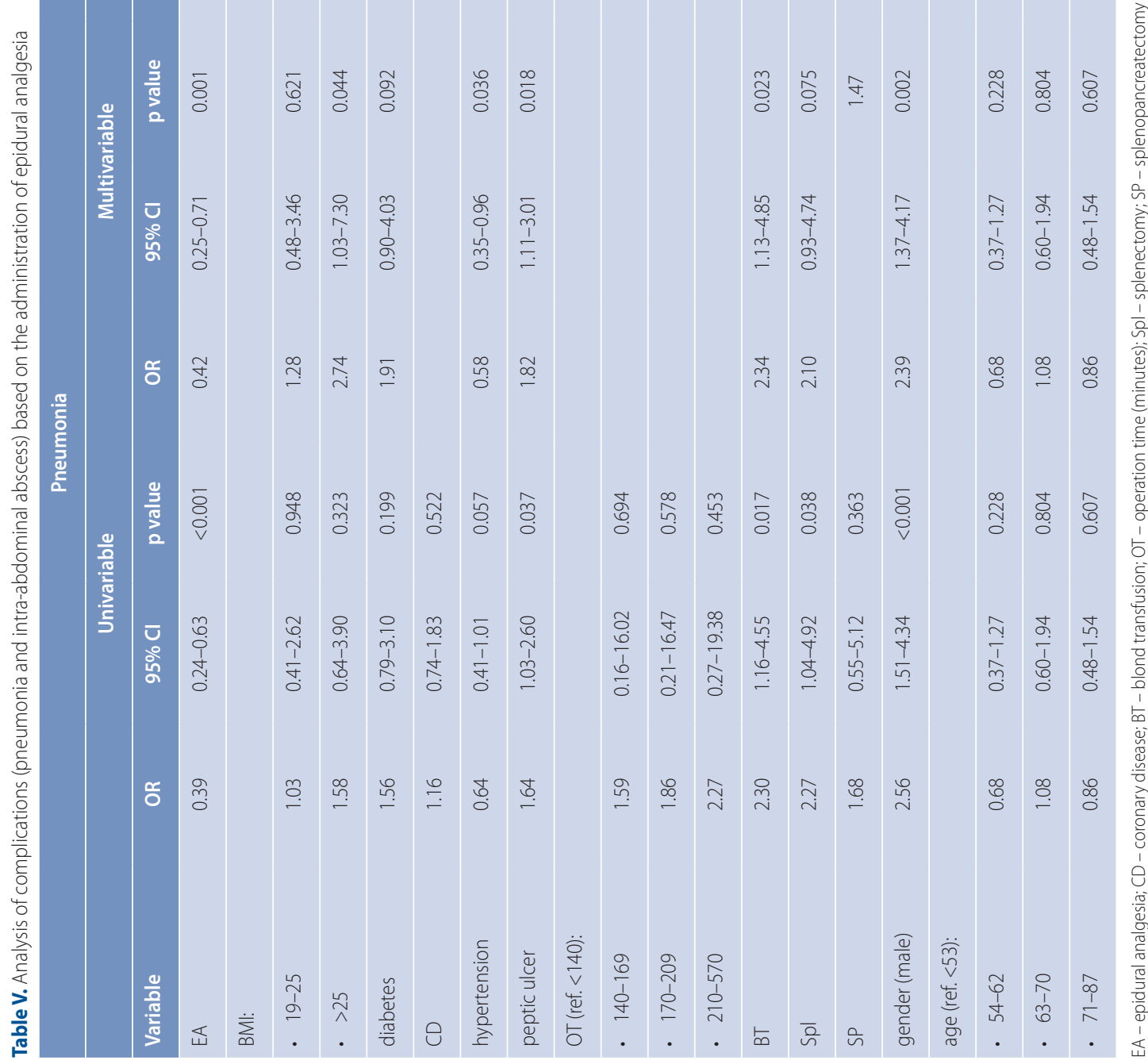




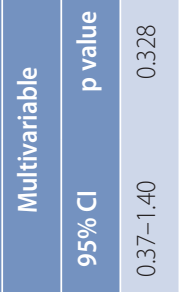

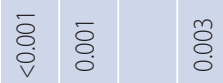

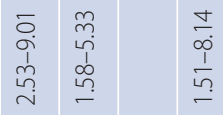

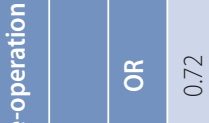

$\underset{+}{\stackrel{8}{*}} \stackrel{\substack{n \\ n}}{i n}$

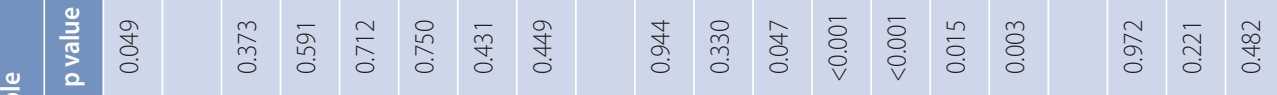

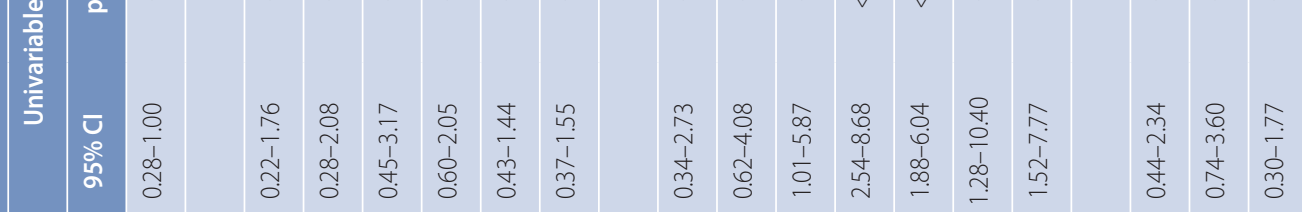

ஊ

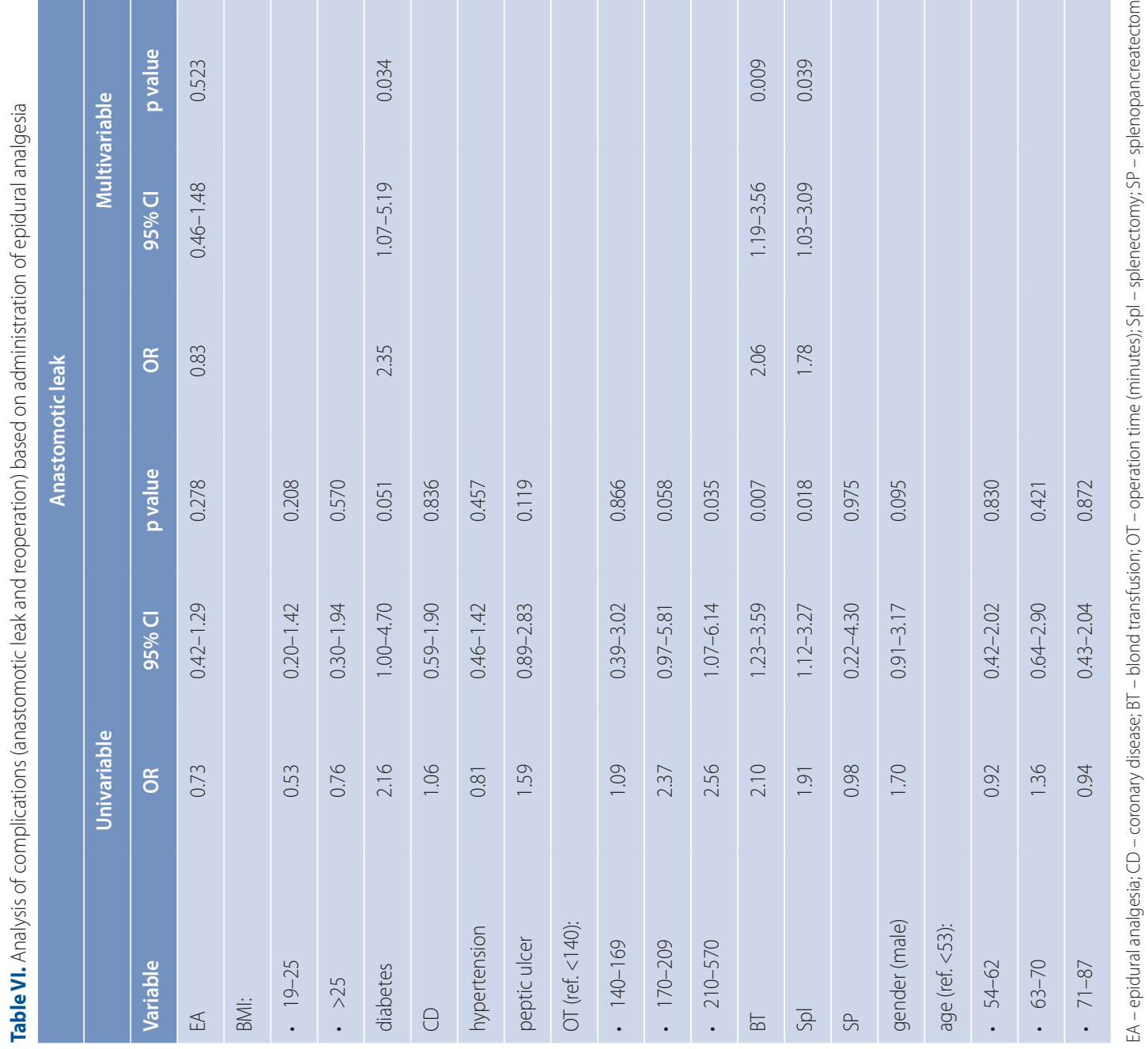




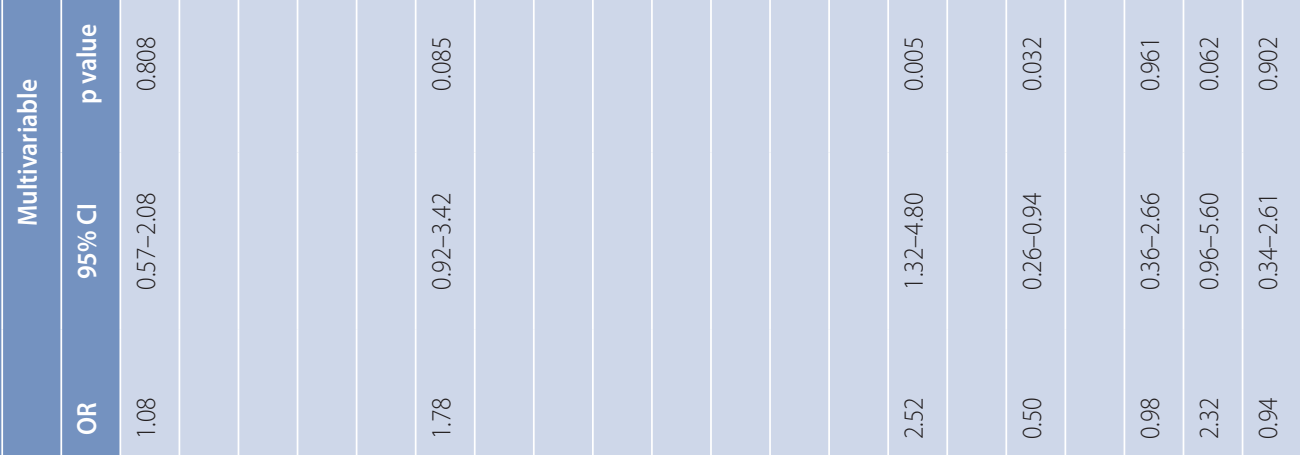

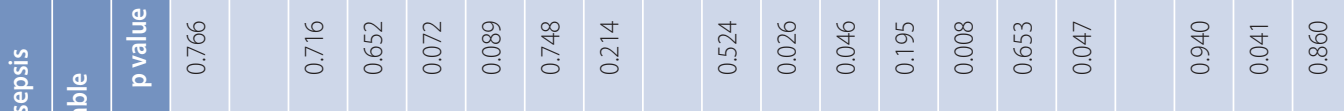

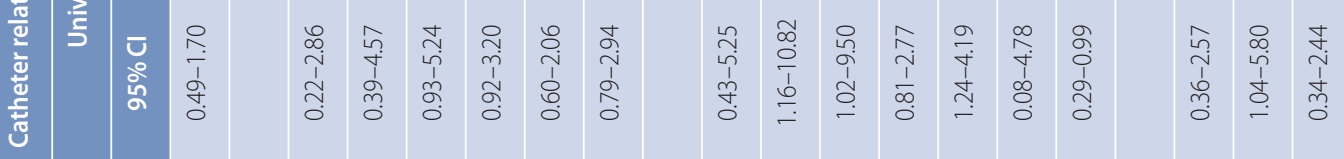

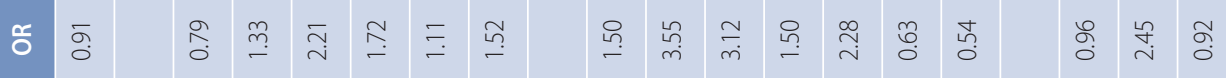

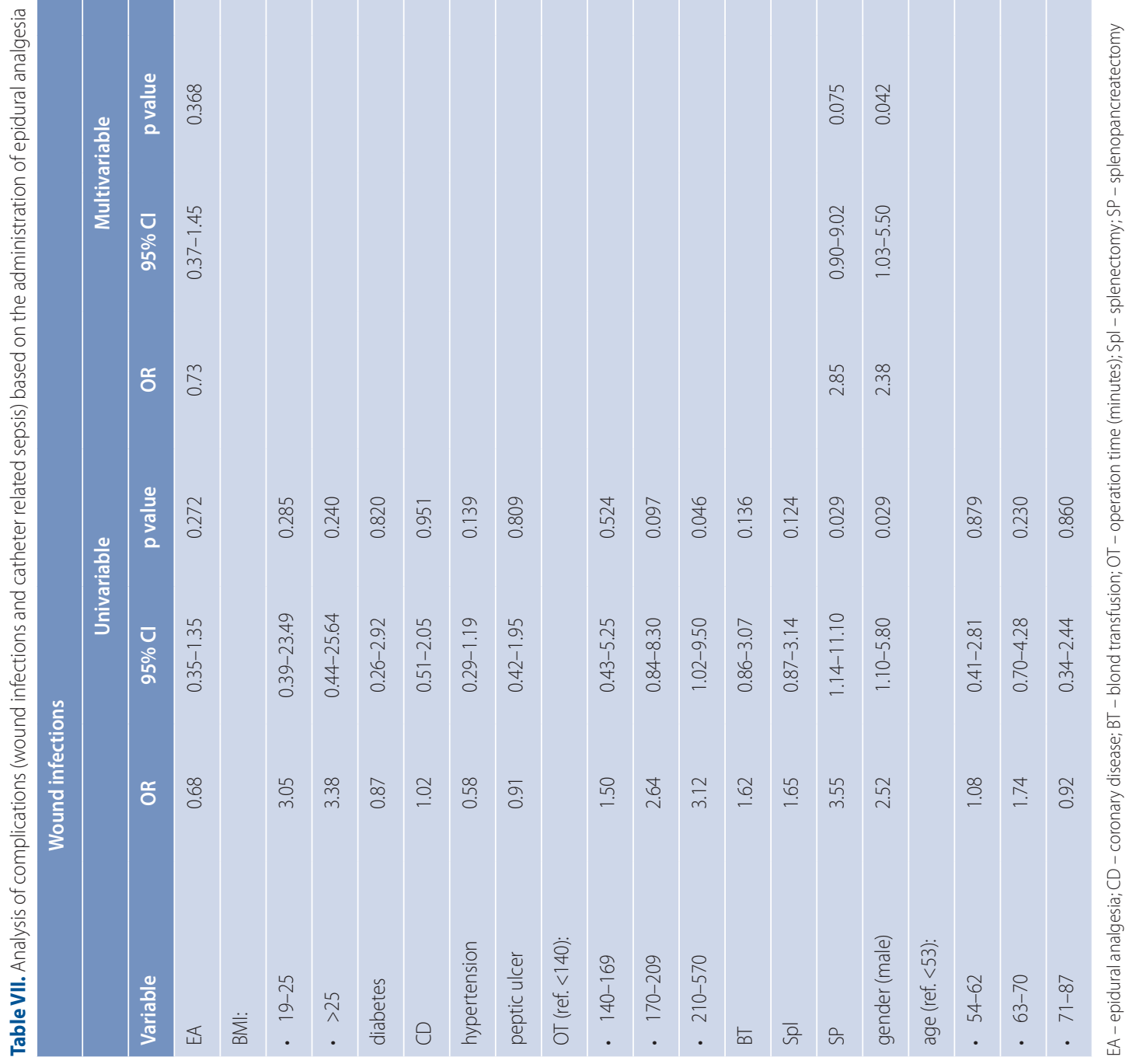


cancer interventions are pneumonia, surgical site infections, (incision infections, intra-abdominal abscesses) and leaking anastomosis [9-14, 18]. Despite the potential for postoperative complications, extensive surgical resection with lymph nodes dissection remains the only curative therapy for gastric cancer worldwide. Experienced medical institutions specializing in surgical oncology routinely perform extensive curative resections for gastric cancer $[9,10,14,19]$.

Effective analgesia is an essential part of postsurgical management and provides statistically and clinically significant improvements in treatment outcomes. Most published clinical studies have demonstrated that the administration of epidural analgesia in gastric surgery patients is a safe practice as a means to improve perioperative outcomes [16, 20-24]. Effective postoperative pain management, as well as the reduction of stress response to surgery along with management of the cardiovascular system and microcirculation significantly reduces complications. Furthermore, studies suggest that administering EA contributes to the reduction of perioperative blood loss. The recommended technique requires continuous infusion of pain medications assisted by intermitted bolus injections $[25,26]$.

A comprehensive literature review of the effect of postoperative analgesia on surgical outcomes [24] showed the impact of administering epidural analgesia on complications rates following major abdominal surgery. Throughout this study, authors established that the administration of epidural analgesia significantly reduces the risk of pulmonary and cardiovascular complications, as well as thromboembolism, postoperative occlusions, and hastens the return of bowel function. Our study was performed retrospectively and is therefore subject to associated biases. During the extensive research period in which the review of this data occurred, our standards of postoperative care and surgical experience have improved; possibly affecting our findings had this data included newer cases. Therefore, based on this study alone, we cannot definitively conclude that administering EA decreases the risk of complications after gastrectomy. There is, however increasing evidence of the overall positive impact that EA has on treatment outcomes. When considering the retrospective review of 84 patients that underwent laparoscopic SG [27], all data suggests that administering EA has no significant impact on treatment outcomes, except for patients treated with EA who experienced urinary retention.

In a prospective study of 1021 patients, the analysis confirmed more effective pain management, a lower need for analgesics, and a shorter stay in the intensive care unit [28]. No statistically significant differences were reported for mortality and the postoperative complication rate. Further analysis demonstrated a reduction of postoperative complications in the group of patients administered EA that underwent vascular surgical interventions. In the relatively smaller groups of patients that underwent gastrectomy (77 patients), large intestine or bile duct operation, the difference between the number of postoperative complications remains insignificant. The results of the Cochrane Database analysis [29] in which 94 studies were evaluated, (total of 5864 patients) suggests effective pain management and an accelerated return of gastrointestinal transit in patients treated with EA. With the use of the open surgery technique, EA reduces the length of the hospital stay. There was no difference in vomiting incidence or anastomotic leak. Complications of epidural analgesia are rare, but additional studies to examine the impact of administering epidural analgesia in extensive surgical interventions for gastric cancer are needed.

A recently publish retrospective review of the American College of Surgeons National Surgical Quality Improvement Program [30] performed for patients undergoing open elective esophagectomies and gastrectomies for nonmetastatic cancer, analyzed a group of 2599 gastrectomies, among which 18\% received $E A$. The only conclusion from the analysis is that EA was associated with a longer length of stay (EA median [IQR] $8[7,11]$ vs. no EA $7[6,11], p=0.0002)$. No other differences between the groups were noted.

Of the retrospective review of 723 gastric cancer resections performed at our institution, 617 cases had complete medical documentation that was adequate for review (85.3\%). Data not included in this study amounting to the remaining $14.7 \%$ of cases was excluded due to random issues such as incomplete medical records and other associated factors. The analyzed group of patients was treated with comparable surgical techniques, postoperative care, and perioperative management protocols. Patients administered EA did not experience a higher number of complications than the group of patients treated with other methods. Thus, administering EA has proven to be safe in the perioperative care of patients undergoing gastric resection. Research indicates that the frequency of wound infections (fever $>38^{\circ} \mathrm{C}$, intra-abdominal abscess) pneumonia and reoperations is reduced in the group of patients with EA. Metaanalysis [26] as well as our assessments confirm that effective postoperative pain management decreases the incidence of pulmonary complications. We observed a decreased number of other complications, (except for frequency of anastomotic leak), however in conclusion, they offer no statistical significance.

\section{Conclusions}

Our findings indicate that administering EA to patients undergoing major stomach resection for gastric cancer is safe. Furthermore, postoperative treatment with epidural analgesia following stomach resection contributes to a reduction in the number of postoperative complications; this is most notable in the reduced number of cases of pneumonia, sepsis, and the need for additional surgical interventions.

\section{Conflict of interest: none declared}




\section{Tomasz Olesińsk}

Maria Sklodowska-Curie National Research Institute of Oncology ul. Roentgena 5

02-781 Warszawa, Poland

e-mail: tolesinski@coi.pl

Received: 20 Mar 2021

Accepted: 23 May 2021

\section{References}

1. Ferlay J, Shin HR, Bray F, et al. GLOBOCAN 2008 v1.2. Cancer Incidence and Mortality Worldwide: IARC Cancer Base No.10. Lyon, France: International Agency for Research on Cancer. 2010. http://globocan.iarc.fr.

2. European Union Network of Excellence (EUNE) for Gastric Cancer Steering Group. Gastric cancer in Europe. Br J Surg. 2008; 95(4): 406-408, doi: 10.1002/bjs.6197, indexed in Pubmed: 18314930.

3. Krajowy rejestr nowotworów. www.onkologia.org.pl.

4. Angelis RDe, Sant M, Coleman M, et al. Cancer survival in Europe 1999-2007 by country and age: results of EUROCARE-5-a population-based study. Lancet Oncol. 2014; 15(1): 23-34, doi: 10.1016/ s1470-2045(13)70546-1.

5. Smyth EC, Verheij M, Allum W, et al. ESMO Guidelines Committee. Gastric cancer: ESMO Clinical Practice Guidelines for diagnosis, treatment and follow-up. Ann Oncol. 2016; 27(suppl 5):v38-v49, doi: 10.1093/annonc/ mdw350, indexed in Pubmed: 27664260.

6. Brar SS, Mahar AL, Helyer LK, et al. Processes of care in the multidisciplinary treatment of gastric cancer: results of a RAND/UCLA expert panel. JAMA Surg. 2014; 149(1): 18-25, doi: 10.1001/jamasurg.2013.3959, indexed in Pubmed: 24225775.

7. Ajani JA, Bentrem DJ, Besh S, et al. National Comprehensive Cancer Network. Gastric cancer, version 2.2013: featured updates to the NCCN Guidelines. J Natl Compr Canc Netw. 2013; 11(5):531-546, doi: 10.6004/ jnccn.2013.0070, indexed in Pubmed: 23667204.

8. Japanese Gastric Cancer Association. Japanese gastric cancer treatment guidelines 2010 (ver. 3). Gastric Cancer. 2011; 14(2): 113-123, doi: 10.1007/s10120-011-0042-4, indexed in Pubmed: 21573742.

9. Sano T, Sasako M, Yamamoto S, et al. Gastric cancer surgery: morbidity and mortality results from a prospective randomized controlled trial comparing D2 and extended para-aortic lymphadenectomy--Japan Clinical Oncology Group study 9501. J Clin Oncol. 2004; 22(14): 2767-2773, doi: 10.1200/JCO.2004.10.184, indexed in Pubmed: 15199090.

10. Wu CW, Hsiung CA, Lo SS, et al. Randomized clinical trial of morbidity after D1 and D3 surgery for gastric cancer. Br J Surg. 2004; 91(3): 283-287, doi: 10.1002/bjs.4433, indexed in Pubmed: 14991627.

11. Memon MA, Subramanya MS, Khan S, et al. Meta-analysis of D1 versus D2 gastrectomy for gastric adenocarcinoma. Ann Surg. 2011; 253(5): 900-911, doi: 10.1097/SLA.0b013e318212bff6, indexed in Pubmed: 21394009.

12. Ichikawa D, Kurioka H, Yamaguchi T, et al. Postoperative complications following gastrectomy for gastric cancer during the last decade. Hepatogastroenterology. 2004; 51(56): 613-617, indexed in Pubmed: 15086217.

13. Cuschieri A, Fayers P, Fielding J, et al. Postoperative morbidity and mortality after D1 and D2 resections for gastric cancer: preliminary results of the MRC randomised controlled surgical trial. The Surgical Cooperative Group. Lancet. 1996; 347(9007): 995-999, doi: 10.1016/ s0140-6736(96)90144-0, indexed in Pubmed: 8606613.

14. Barchi LC, Charruf AZ, de Oliveira RJ, et al. Management of postoperative complications of lymphadenectomy. TransI Gastroenterol Hepatol. 2016; 1: 92, doi: 10.21037/tgh.2016.12.05, indexed in Pubmed: 28138657.

15. Yamada T, Hayashi T, Cho $\mathrm{H}$, et al. Usefulness of enhanced recovery after surgery protocol as compared with conventional perioperative care in gastric surgery. Gastric Cancer. 2012; 15(1):34-41, doi: 10.1007/ s10120-011-0057-x, indexed in Pubmed: 21573918.

16. Sugisawa N, Tokunaga M, Makuuchi R, et al. A phase II study of an enhanced recovery after surgery protocol in gastric cancer surgery. Gastric Cancer. 2016; 19(3): 961-967, doi: 10.1007/s10120-015-0528-6, indexed in Pubmed: 26260875.

17. Niemi G, Breivik H. Adrenaline markedly improves thoracic epidural analgesia produced by a low-dose infusion of bupivacaine, fentanyl and adrenaline after major surgery. A randomised, double-blind, cross-over study with and without adrenaline. Acta Anaesthesiol Scand. 1998; 42(8): 897-909, doi: 10.1111/j.1399-6576.1998.tb05348.x, indexed in Pubmed: 9773133.

18. Gil-Rendo A, Hernández-Lizoain JL, Martínez-Regueira F, et al. Risk factors related to operative morbidity in patients undergoing gastrectomy for gastric cancer. Clin TransI Oncol. 2006; 8(5): 354-361, doi: 10.1007/ s12094-006-0182-x, indexed in Pubmed: 16760011.

19. Songun I, Putter $\mathrm{H}$, Kranenbarg EMK, et al. Surgical treatment of gastric cancer: 15-year follow-up results of the randomised nationwide Dutch D1D2 trial. Lancet Oncol. 2010; 11(5): 439-449, doi: 10.1016/S1470-2045(10)70070-X, indexed in Pubmed: 20409751.

20. Liu S, Carpenter RL, Neal JM. Epidural anesthesia and analgesia. Their role in postoperative outcome. Anesthesiology. 1995; 82(6): 1474-1506, doi: 10.1097/00000542-199506000-00019, indexed in Pubmed: 7793661.

21. Harukuni I, Yamaguchi $H$, Sato $S$, et al. The comparison of epidura fentanyl, epidural lidocaine, and intravenous fentanyl in patients undergoing gastrectomy. Anesth Analg. 1995; 81(6): 1169-1174, doi: 10.1097/00000539-199512000-00009, indexed in Pubmed: 7486099.

22. Wu Y, Liu F, Tang $\mathrm{H}$, et al. The analgesic efficacy of subcostal transversus abdominis plane block compared with thoracic epidural analgesia and intravenous opioid analgesia after radical gastrectomy. Anesth Analg. 2013; 117(2): 507-513, doi: 10.1213/ANE.0b013e318297fcee, indexed in Pubmed: 23744953.

23. Wheatley RG, Schug SA, Watson D. Safety and efficacy of postoperative epidural analgesia. Br J Anaesth. 2001; 87(1): 47-61, doi: 10.1093/ $\mathrm{bja} / 87.1 .47$.

24. Holte $\mathrm{K}$, Kehlet $\mathrm{H}$, Kehlet $\mathrm{H}$, et al. Effect of postoperative analgesia on surgical outcome. Br J Anaesth. 2001; 87(1): 62-72, doi: 10.1093/ bja/87.1.62, indexed in Pubmed: 11460814.

25. Komatsu $\mathrm{H}$, Matsumoto $\mathrm{S}$, Mitsuhata $\mathrm{H}$, et al. Comparison of patient-controlled epidural analgesia with and without background infusion after gastrectomy. Anesth Analg. 1998; 87(4): 907-910, doi: 10.1097/00000539-199810000-00030, indexed in Pubmed: 9768792.

26. Ballantyne JC, Carr DB, deFerranti S, et al. The comparative effects of postoperative analgesic therapies on pulmonary outcome: cumulative meta-analyses of randomized, controlled trials. Anesth Analg. 1998; 86(3): 598-612, doi: 10.1097/00000539-199803000-00032, indexed in Pubmed: 9495424

27. Yanagimoto $Y$, Takiguchi S, Miyazaki Y, et al. Comparison of pain management after laparoscopic distal gastrectomy with and without epidural analgesia. Surg Today. 2016; 46(2): 229-234, doi: 10.1007/ s00595-015-1162-y, indexed in Pubmed: 25861994.

28. ParkWY, Thompson JS, Lee KK. Effect of epidural anesthesia and analgesia on perioperative outcome: a randomized, controlled Veterans Affairs cooperative study. Ann Surg. 2001; 234(4): 560-9; discussion 569, doi: 10.1097/00000658-200110000-00015, indexed in Pubmed: 11573049.

29. Guay J, Nishimori M, Kopp SL, et al. Epidural local anaesthetics versus opioid-based analgesic regimens for postoperative gastrointestinal paralysis, vomiting and pain after abdominal surgery. Cochrane Database Syst Rev. 2016; 7(6): CD001893-1602, doi: 10.1002/14651858. CD001893.pub2, indexed in Pubmed: 27419911.

30. Pesco J, Young K, Nealon K, et al. Use and Outcomes of Epidural Analgesia in Upper Gastrointestinal Tract Cancer Resections. J Surg Res. 2021; 257: 433-441, doi: 10.1016/j.jss.2020.08.018, indexed in Pubmed: 32892142. 